Experiencia educativa

\title{
Proyecto Persevera: Una respuesta desde la Universidad Don Bosco
}

\author{
Persevere Project: an answer coming from Universidad Don Bosco \\ Yesenia Xiomara Martínez * \\ ymartinez@udb.edu.sv
}

ISSN 1996-1642, Editorial Universidad Don Bosco, año 8, No.13, Enero-Junio de 2014, pp.41-51

Recibido: 5 de enero de 2014. Aceptado: 17 de febrero de 2014

Resumen

La Universidad Don Bosco (UDB), consciente de la responsabilidad por mejorar los indicadores de rendimiento interno implementa Persevera para la reducción de los índices de repitencia y deserción. Dicho programa responde al principio de educar y promover el desarrollo integral de la persona, siendo una respuesta desde los principios pedagógicos del sistema preventivo salesiano que permite la construcción de la síntesis personal de fe, cultura y vida'. Posee cuatro componentes: Observatorio de la repitencia y deserción, Coaching para estudiantes y docentes, Autoformación permanente por medio de recursos físicos y TIC y Estudio de la clase entre los docentes. Aquí se presenta una descripción de cómo se implementó institucionalmente y de sus logros.

Palabras clave: Deserción, repitencia, persevera.
Abstract:

Universidad Don Bosco, taking into consideration its responsibility to improve the academic outcomes, implements the Persevere program in order to lower the repetition and dropout rates. Such program is based on the premise of educating and developing the person as a whole under the pedagogical principle of the Salesian preventing system which enables the building of the personal faith, culture and life. The program has four components: the repetition and dropout observatory, coaching for students and professors, permanent autonomous learning and class study among professors. In this paper the author recalls how the program was implemented and some of its achievements.

Keywords: repetition, dropouts, persevere.

\footnotetext{
* La autora es Maestra en gestión de la calidad y Secretaria General de la Universidad Don Bosco.

1. Persevera también responde al estilo de educador salesiano que se caracteriza por: la sensibilidad social; el protagonismo y el diálogo entre los diversos actores; la presencia cercana del educador, que es facilitador y animador; la dimensión comunitaria del hecho educativo; el estilo cooperativo de aprendizaje; el reconocimiento y valoración de las diferencias culturales así como la capacidad de promover el diálogo intercultural; el espíritu crítico, propositivo y emprendedor; la vinculación con la vida y la articulación entre teoría y práctica, entre educación y mundo del trabajo; la búsqueda honesta de la verdad mediante la investigación; una visión optimista del mundo y de la historia; la integralidad de las propuestas educativas (Fundamentos Institucionales Universidad Don Bosco, 2010).
}

Para citar este artículo: Martínez, Y. X. (2014). Programa Persevera: Una respuesta desde la Universidad Don Bosco. Diálogos 13, 41-51. 


\section{Fundamentos teóricos}

"La repitencia y la deserción pueden constituirse en fenómenos colectivos e incluso masivos, y ser estudiados como tales. En dicho caso, por lo general, se asocian a la eficiencia del sistema" (Fiegehen, 2006, p. 157). No basta con realizar una medición de los indicadores de eficiencia interna y realizar un reporte sobre los mismos, esta información es útil siempre y cuando se tomen las acciones necesarias para mejorar la retención y promoción en el nivel superior, teniendo la claridad que la problemática es compleja y como tal demanda soluciones integrales.

Para mejorar los mecanismos de detección temprana, identificar grupos de riesgo, otorgar apoyo tutorial integral al estudiante, mejorar la orientación vocacional, realizar seguimiento estudiantil y mejorar la administración curricular, entregar certificaciones tempranas y salidas intermedias, es necesario tener claridad sobre las causas de los fenómenos en cuestión.

Las causas de la deserción y repitencia, según Fiegehen (2006), están asociadas a cuatro causas principales: externas, del sistema e institución, de tipo académico y de tipo personal².

Además, la falta de sistemas de información fiables que permitan diferenciar cuando un estudiante abandona sus estudios y cuando se inscribe en otro curso o en otra universidad, dificulta contar con estimaciones precisas. "En algunos casos, afirma Gazzola (2008, p. 331), "se ha analizado la tasa de graduación oportuna (...)". Los estudios sobre la graduación oportuna deben tener en cuenta que en la mayoría de los sistemas universitarios de América Latina una alta proporción de estudiantes no dedican un tiempo completo a sus estudios. Esto explica, en gran medida, la prolongación de los mismos más allá del tiempo teórico. Nos introducimos por tanto en el ámbito de las implicaciones de la deserción y la repitencia que pueden ser de tres tipos: sociales, institucionales y personales ${ }^{3}$ (Fiegehen, 2006). No obstante, son pocos los países que han desarrollado programas específicos para responder a este problema a nivel de

2. Externas (Las condiciones socioeconómicas tanto del estudiante como del grupo familiar), del Sistema e Institucionales (Carencia de mecanismos adecuados de financiamiento del sistema en especial para el otorgamiento de ayudas estudiantiles, créditos y becas, el desconocimiento de la profesión y de la metodología de las carreras; el ambiente educativo e institucional y la carencia de lazos afectivos con la universidad), de tipo_académico (Formación académica previa, los exámenes de ingreso, el nivel de aprendizaje adquirido, la falta de apoyo y orientación recibida por los profesores, la falta de información al elegir la carrera; la carencia de preparación para el aprendizaje y reflexión autónoma) y personales (Aspectos de orden tanto motivacionales como actitudinales) (Fiegehen, 2006).

3. Sociales (La retroalimentación del circulo de la pobreza y la gestación de una "capa social" de frustrados profesionales, con posible disminución del aporte intelectual y el potencial aumento del subempleo, Institucionales (La limitación para cumplir la misión institucional y un descenso en los índices de eficiencia y calidad y Personales (El disgusto, la frustración y la sensación de fracaso de los repitentes y desertores con los consiguientes efectos en su salud física y mental (Fiegehen, 2006). 
sistema. Dos ejemplos son Colombia con su Sistema para la Prevención y Análisis de la Deserción en las Instituciones de Educación Superior (SPADIES) ${ }^{4}$, por medio del cual se identifican y se ponderan los comportamientos, las causas, variables y riesgos determinantes para desertar; y México con su Programa Nacional de Becas para la Educación Superior (PRONABES) ${ }^{5}$, mediante el cual estudiantes en situación económica adversa y con deseos de superación pueden continuar en el nivel superior en instituciones públicas en programas de licenciatura o de técnico superior universitario, reduciendo con ello el riesgo de abandono por factores económicos.

En El Salvador, como en otros países, las universidades requerirán desarrollar mecanismos para acompañar a los estudiantes de mayor riesgo para aumentar sus posibilidades de éxito académico (Gazzola, 2008).

\section{Una propuesta para la reducción de la repitencia y la deserción}

En el Informe de la Educación Superior en América Latina y el Caribe 2000-2005 (2006) "La metamorfosis de la educación superior" de la UNESCO, se afirma que la deserción escolar o la reprobación en las universidades está provocando que aumenten las afecciones en la salud física y mental de los jóvenes que abandonan la universidad, pero sobre todo representan un elevado costo para los países, ya que se estima que al año en América Latina y el Caribe se pierden entre 2 y 415 millones de dólares por país, producto del abandono o la deserción de los estudios universitarios.

Según los resultados de la Información Estadística de Instituciones de Educación Superior presentado por el Ministerio de Educación de El Salvador (Resultados de la Información Estadística de Instituciones de Educación Superior, 2012), los datos de matrícula en las instituciones de educación superior en el año 2011 han experimentado un crecimiento sostenido, lo cual resulta positivo en función de mejorar el indicador de cobertura en el nivel terciario. La matrícula estudiantil que las instituciones del nivel superior reportaron fue de 160,374 lo cual representa un incremento del 6.91 \% con relación al 2010.

En el año 2011 , la distribución de la matrícula estudiantil que realizó estudios en el nivel terciario según el tipo de institución, se dio de la siguiente manera: las universidades inscribieron a 147,311 estudiantes representando el $91.86 \%$ del

4. El SPADIES fue diseñado por el Centro de Estudios Económicos (CEDE), de la Universidad de los Andes, y está articulado con el Sistema Nacional de Información de la Educación Superior (SNIES), el Instituto Colombiano para el Fomento de la Educación Superior (ICFES) y el Instituto Colombiano de Crédito Educativo y Estudios Técnicos en el Exterior (ICETEX).

5. PRONABES tuvo sus inicios en el ciclo escolar 2001-2002 con la participación de todas las Entidades Federativas y la de cuatro instituciones. públicas de educación superior federales, -IPN, UAM, UNAM, y UPN-. Los recursos del fondo son aportados por el Gobierno Federal, los gobiernos estatales y las instituciones públicas de educación superior federales por partes iguales. 
total de la población en el nivel superior, los institutos especializados tuvieron una matrícula de 8,776 estudiantes significando el 5.47\% y los institutos tecnológicos recibieron a 4,287 estudiantes que representa el $2.67 \%^{\circ}$ (Resultados de la Información Estadística de Instituciones de Educación Superior, 2012)

De los factores más relevantes que inciden en la deserción se tienen: bajos recursos económicos que obliga a los jóvenes a buscar un empleo, la falta de orientación respecto a los programas y al mercado profesional, el cambio de situación familiar de solteros(as) a casados(as).

Por otro lado, los indicadores que integran esta categoría, son los más difíciles de obtener y abarcan los problemas más graves de los sistemas educativos de nuestros países. Cada vez que se habla de eficiencia interna se hace referencia a la repetición, deserción y otras variables asociadas. Los expertos opinan que repetidores y desertores son los educandos más caros que pagan las sociedades, se invierte en ellos y no se obtienen resultados positivos.

Los indicadores de eficiencia considerados trazadores son los años promedio que los alumnos permanecen en el sistema, número de años promedio para completar cada nivel y porcentaje de alumnos que se gradúan. El presente escrito tiene por objetivo dimensionar la magnitud de la Deserción y la Repitencia en las carreras de la Universidad Don Bosco, analizando los factores que inciden en ellas, sus implicaciones y posibles propuestas para paliarlas.

La Universidad Don Bosco de El Salvador, consciente de la enorme responsabilidad que se tiene para enfrentar estos desafíos, ha diseñado una propuesta para la reducción de la repitencia y deserción, denominado Programa Persevera, mejorando los índices de eficiencia interna.

El Programa Persevera es ante todo un llamado a los estudiantes para que practiquen la perseverancia, la constancia, el esfuerzo permanente, a ser firme en sus actuaciones Además, al ser generado en un ambiente salesiano, el programa también demanda del acompañamiento, de la asistencia, de la presencia constante de los educadores. Juntos, estudiantes y educadores, se desafíen al establecer metas académicas desde una visión integral del ser humano y desde el estilo salesiano, es decir, con trabajo, templanza y alegría.

Esto significa, entre muchas otras cosas, dar una respuesta concreta desde el ámbito pedagógico para

Incrementar la autoestima y autoconocimiento; crear redes de apoyo; trabajar la motivación y autodeterminación; perfeccionar los procesos 
cognitivosymetacognitivos; incorporarel manejoy control de la ansiedad; considerar los estilos de aprendizaje, la atención, la concentración y los distintos tipos de inteligencias; incorporar sistemas de nivelación y remediales, el establecimiento de ciclos generales de conocimientos básicos, generar condiciones adecuadas para el aprendizaje (métodos, infraestructura y recursos); establecer innovaciones curriculares (perfiles y enseñanza por competencias y fortalecer la metodología de resolución de problemas); establecer currículos más flexibles; establecer una titulación directa con un trabajo de investigación desarrollado en el último año; realizar cambios metodológicos; incorporar TIC; lograr el perfeccionamiento pedagógico de los docentes y mejorar los procesos de evaluación; la detección temprana de posibles desertores, prevenir y darles el apoyo necesario y determinar los momentos problemáticos. Informar y orientar sobre las distintas ramas científicas, con énfasis en aspectos de destrezas requeridas así como en las oportunidades del mercado profesional; acordar convenios con sectores productivos para prácticas de internado y para la realización de investigaciones; establecer un diseño y administración curricular apropiado; generar procesos administrativos eficientes y dar orientación al estudiante con riesgos de desertar (Fiegehen, 2006, p. 165).

\section{Fundamentos pedagógicos salesianos}

De acuerdo al ideario de la Universidad Don Bosco, Persevera responde al principio de educar y promover el desarrollo integral de la persona humana; contribuyendo con estilo salesiano a la construcción, en la verdad, de una sociedad libre, justa y solidaria. Todo ello desde una perspectiva cristiana y humanista. Por ello, es una respuesta desde los principios pedagógicos del sistema preventivo de Don Bosco (Razón-Religión-Amorevolezza) que permiten la construcción de la síntesis personal de fe, cultura y vida.

Es decir, se fundamenta en la convicción de que la persona se va configurando cotidianamente y se autoposee en cuanto asume la búsqueda de la verdad como misión y tarea; en cuanto es capaz de elegir libremente unos valores que den sentido a su existencia humana; y en la medida en que se hace protagonista de la historia participando responsablemente en el desarrollo de su comunidad. La persona se autocomprende como un ser de posibilidades cuando las reconoce y las desarrolla" (Ideario Universidad Din Bosco, 2010).

Persevera también responde al estilo de educar salesiano que se caracteriza por la sensibilidad social; el protagonismo y el diálogo entre los diversos actores; la presencia cercana del educador, que es facilitador y animador; la dimensión comunitaria del hecho educativo; el estilo cooperativo de aprendizaje; el 
reconocimiento y valoración de las diferencias culturales así como la capacidad de promover el diálogo intercultural; el espíritu crítico, propositivo y emprendedor; la vinculación con la vida y la articulación entre teoría y práctica, entre educación y mundo del trabajo; la búsqueda honesta de la verdad mediante la investigación; una visión optimista del mundo y de la historia; la integralidad de las propuestas educativas (Fundamentos Institucionales UDB (2010).

\section{Componentes del Programa Persevera}

Persevera tiene cuatro componentes (ver Ilustración 1) que interactúan entre sí para aumentar la promoción y la supervivencia escolar, así como el perfeccionamiento docente, en la Universidad Don Bosco:

1. Observatorio de la Repitencia y Deserción. Monitoreo permanente de los indicadores en cuestión haciendo uso del Gestor Académico ${ }^{7}$ y otras bases de datos e informes con que cuenta la UDB como son: informe de caracterización socioeconómica, informes de atención psicopedagógica, entre otros.

2. Coaching 8 para estudiantes y docentes. Formación de coach (técnicos y docentes) e identificación de coachee (estudiantes) que estén en situación de repitencia $o$ en riesgo de abandono del sistema, para desarrollarles sus capacidades de acción y establecimiento de compromisos personales integrales.

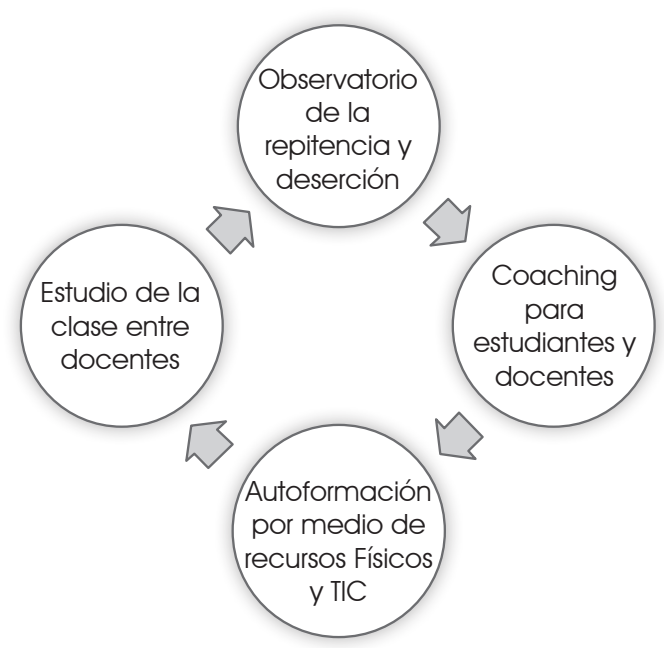

Ilustración 1: Componentes del Programa Persevera 
3. Autoformación permanente por medio de recursos físicos y TIC. Refuerzo académico e identificación de bibliografía ad hoc para su reproducción física y electrónica (web) que fomenten la autodisciplina y el apoyo mutuo en equipos de trabajo y redes virtuales. Además incluye un fondo de becas para estudiantes con rezago académico por razones económicas.

4. Estudio de la clase. Método con el que el personal docente realiza la mejora de la enseñanza en clase por medio de la observación e investigación, reflexión conjunta, mejora de los materiales didácticos y recursos evaluativos. Con ello se cultiva su propia capacidad y confianza en sí mismo.

\section{Resumen de los principales resultados}

El programa Persevera ha sido implementado en la UDB desde al año 2012. Como resultados globales se pueden mencionar que en la UDB en los últimos 5 años presenta una tendencia positiva en su matrícula, y en términos generales la deserción general en la UDB se ha venido reduciendo, todo esto con las diferentes estrategias que se han aplicado en la institución. Por tanto, Persevera al ser un programa multifuncional e integrador poco a poco persigue lograr empoderar a los estudiantes como protagonistas de su ingreso, permanencia y egreso de la vida universitaria, de tal forma que su identidad y capacidades se vean fortalecidas para un desarrollo exitoso de sus competencias dentro de la carrera que responde a sus más profundas aspiraciones de vida.

Persevera no sólo es el hecho de hacer coaching, sino que le permite a la UDB obtener un diagnóstico de la fuente primaria9 que vive el problema, por medio de los informes semestrales que facilita cada coach sobre la problemática de la deserción y la repitencia en los jóvenes universitarios abordados por medio de las sesiones, obteniendo de esta manera insumos para la toma de acciones no solo desde el Programa Persevera, sino desde otras instancias.

Algunos avances, en cada uno de sus componentes, son los siguientes:

\subsection{Observatorio de la repitencia y deserción}

Se realiza el monitoreo permanente de los indicadores de repitencia y deserción. Los indicadores analizados son: matrícula de nuevo y antiguo ingreso, la deserción y la repitencia. En términos de deserción se ha logrado una disminución en los últimos años, desde el 2005 (14.03\%) al 2013 (11.2\%), no obstante, no son suficientes. Por su parte, la repitencia en la UDB indica que 23 de cada 100 estudiantes están cursado al menos una asignatura en $2^{a}$ matrícula.

9. Los mismos estudiantes y sus experiencias durante el proceso en el programa Persevera que se benefician del acompañamiento salesiano 
La Universidad ha realizado acciones para disminuir los porcentajes de reprobación que incluyen: asesorías, coaching, tutorías, sesiones y talleres de atención psicopedagógica, círculos de estudio; sin embargo, es importante identificar desde la percepción de los estudiantes y profesores las causas que generan la reprobación.

\subsection{Coaching para estudiantes y docentes}

Se han realizado tres formaciones en el tema de coaching para los docentes de las diferentes facultades haciendo un total de 70 profesionales de la Universidad, de estos 45 han realizado coaching con los estudiantes (coachees) que les han sido asignados.

El perfil de los coachees seleccionados es (a) estudiantes de nuevo ingreso que estén en riesgo de repitencia, teniendo en cuenta número de asignaturas y tipo de asignaturas reprobadas; (b) estudiantes de antiguo ingreso que reprobaron asignaturas, y necesitan seguimiento de su proceso, tomando en cuenta: $\mathrm{CUM}^{10}$, número de asignaturas inscritas en el semestre y tipo de asignaturas reprobadas.

Con cada estudiante-coachee seleccionado se realizan al menos tres sesiones por semestre con el objetivo de brindar seguimiento al proceso del estudiante y acompañarlo para potenciar al máximo sus capacidades, superando por él mismo cualquier dificultad que se le presente ya sea personal o académica.

Se tiene definido que cada docente que realiza sesiones de coaching atiende a un máximo de 10 estudiantes por semestre.

Es importante mencionar que a través de estas sesiones se puede evidenciar que cada uno de los estudiantes, tiene diferentes situaciones que afectan su rendimiento académico y su motivación o dedicación.

Algunas de las causas de reprobación que se han identificado durante la implementación del programa son las siguientes:

1. Nivel bajo de madurez personal de los estudiantes debido a su corta edad, nivel de desarrollo y madurez con la que ingresan a la universidad, lo que provoca su falta de interés y compromiso con el estudio.

2.Bajo perfil de ingreso de los estudiantes de acuerdo con las pruebas diagnósticas y psicopedagógicas realizadas por la universidad. 
3. Inexistentes o deficientes hábitos de estudio, y poca disciplina para organizar el tiempo.

4. Una dependencia al uso del internet y teléfonos inteligentes para el uso de redes sociales y entretenimiento, lo que provoca la falta de concentración en la realidad.

5.Problemas al interior de la vida familiar, y la dificultad para enfrentarlos y sobreponerse a ellos (económicos, violencia, trabajo, hacinamiento, falta de espacios para estudiar)

6.Problemas al interior de las familias, relaciones conflictivas, responsabilidades asignadas por negocios familiares, contexto familiar con mucho ruido y poco espacio.

7. Condición económica de los estudiantes lo que les obliga a buscar un empleo para poder seguir estudiando.

8. Nivel de seguridad en el país, lo que atemoriza salir de sus hogares.

9. Mala distribución de la carga académica asignada a un estudiante de nuevo ingreso, en algunas carreras se lleva hasta 6 asignaturas en el primer ciclo.

A año y medio de su ejecución se puede observar los siguientes resultados:

1. En el año 2012, en el primer semestre que se implementó Persevera, del total de estudiantes que fueron convocados a las sesiones de coaching asistió el 40,34\%, de estos, el 30,21\% mejoró en su rendimiento académico. En el segundo semestre asistió a las sesiones de coaching el $48.0 \%$, de estos el $47 \%$ mejoró en su rendimiento académico. En suma para ambos semestres se asignó a un total de 325 estudiantes a los primeros 45 docentes que fueron formados en coaching.

2. Previo a las sesiones de coaching se han implementado diferentes acciones de acompañamiento para los estudiantes, las cuales dan inicio desde el momento en que el estudiante ingresa a la Universidad, por medio del Curso de Inducción de la Vida Universitaria (CIVU), donde los estudiantes reciben orientación vocacional, realizan pruebas diagnósticas y psicopedagógicas, participan en talleres acordes a la carrera y reciben asesoría académica previa al inicio del ciclo correspondiente.

3. Los estudiantes que asistieron a las sesiones de coaching manifiestan que les ha ayudado mucho en su proceso de adaptación a la Universidad.

4. Identificación de casos especiales estudiantes-coachees para referirlos al Departamento de Atención Psicopedagógica (DAC).

5. Generación de valores como la confianza entre Coach y Coachee para abordar diversidad de aspectos no solo de la academia, si no personales, para ayudarle a desenvolverse de manera eficiente en el proceso de enseñanza-aprendizaje. 


\subsection{Autoformación permanente por medio de recursos físicos y TIC}

Se ha iniciado con una plataforma interna en la que se colocan recursos electrónicos que ayudan a la autoformación de los estudiantes, adicional a esto la Universidad pone a disposición de los estudiantes la Tutoría Estudiantil: la cual es una actividad de acompañamiento que tiene como objetivo orientar y apoyar a los alumnos durante su proceso de formación. La tutoría es parte del estilo salesiano de la Universidad Don Bosco, cuya importancia radica en orientar a los alumnos a partir del conocimiento de sus problemas y necesidades académicas, así como de sus inquietudes y aspiraciones profesionales.

La Universidad es consciente que buena parte de los jóvenes que ingresan a la UDB, se encuentran en situaciones difíciles, y por eso coloca a disposición de ellos diferentes programas de apoyo, considerando también que existen factores externos en cada estudiante que afectan su rendimiento académico como: situación económica, bases académicas previas deficientes, hábitos de estudios deficientes, situación social y familiar, entre otras. Para esto se gestionan fondos de becas para aquellos estudiantes que se encuentren en riesgo de deserción debido a problemas económicos.

\subsection{Estudio de la clase}

Finalmente en el componente que se refiere al Estudio de la clase, el Instituto de Investigación y Formación Pedagógica de la Universidad, ha iniciado en el semestre del año 2013 estudios de aquellas asignaturas que tienen mayores índices de reprobación, con el objetivo de tener la evaluación y la mejora de las estrategias metodológicas que actualmente se utilizan.

\section{Reflexión sobre las implicaciones teóricas, prácticas o metodológicas}

De acuerdo con los primeros resultados, a un año de su implementación, se identifican que las causas de deserción y repitencia efectivamente están relacionadas a las cuatro causas principales que menciona Fiegehen (2006): Causas externas como las condiciones socioeconómicas tanto del estudiante como del grupo familiar, las Institucionales como la carga académica de los estudiantes, la falta de un programa de becas con mayor cobertura, de tipo académico como el bajo perfil de ingreso de los estudiantes, malos hábitos de estudio y falta de acompañamiento personalizado de los docentes, y las causas personales como la falta de interés y compromiso con el estudio.

A pesar de contar ya con un sistema de información fiable (Sistema Informático, Gestor Académico), que cuenta con toda la información académica y personal 
del estudiante todavía hace falta la cultura de los docentes para acudir a él y acompañar con mayor eficiencia a los estudiantes como afirma Gazzola (2008).

De acuerdo con el ideario de la Universidad Don Bosco, el Programa Persevera responde al principio de educar y promover el desarrollo integral de la persona humana. Por ello, Persevera es una respuesta desde los principios pedagógicos del sistema preventivo de Don Bosco (Razón-Religión-Amorevolezza) que permiten la construcción de la síntesis personal de fe, cultura y vida. PERSEVERA también responde al estilo de educar salesiano que se caracteriza por la sensibilidad social; el protagonismo y el diálogo entre los diversos actores; la presencia cercana del educador, que es facilitador y animador; la dimensión comunitaria del hecho educativo; el estilo cooperativo de aprendizaje; el reconocimiento y valoración de las diferencias culturales así como la capacidad de promover el diálogo intercultural; el espíritu crítico, propositivo y emprendedor; la vinculación con la vida y la articulación entre teoría y práctica, entre educación y mundo del trabajo; la búsqueda honesta de la verdad mediante la investigación; una visión optimista del mundo y de la historia; la integralidad de las propuestas educativas (Fundamentos Institucionales UDB, 2010).

\section{Conclusiones}

El programa Persevera es una opción válida para enfrentar el problema de la deserción y la repitencia, ya que los jóvenes necesitan siempre una guía que les ayude a descubrir sus competencias y aptitudes para la vida universitaria, y poder de esta forma tomar decisiones por sí mismos en lo referente a su desarrollo profesional y social.

Es importante establecer estrategias para la disminución de la deserción que generen la conciencia en los estudiantes para ser mejores en su rendimiento académico y personal, y para eso el coaching en una excelente herramienta.

La observación de clases de los docentes por expertos pedagogos y técnicos apoya a que se establezcan nuevas estrategias metodológicas, que deben ser usadas por los docentes, ante la nueva generación de estudiantes que ingresan a las universidades.

\section{Referencias}

Fundamentos Institucionales l: proyecto de innovación curricular:modelo 
educativo, ideario, misión, visión. (2010). San Salvador: Universidad Don Bosco.

Gazzola A. L. (2008). Tendencias de la Educación Superior en América Latina y el Caribe. Caracas, Venezuela: IESALC-UNESCO.

Colombia, M. d. (s.f.). SPADIES Ministerio de Educación Nacional de Colombia. Recuperado el 2013, de http://www.mineducacion.gov.co/1621/w3article-156292.html

Fiegehen, L. E. (2002). Repitencia y deserción universitaria en América Latina. En I. I. (IESALC), Informe sobre la Educación Superior de América Latina y el Caribe 2002-2005. La metamorfosis de la Educación Superior (págs. 156-166). Caracas: Metropolis.

Programa Nacional de Becas para la Educación Superior (s.f.). Recuperado el 2013, de http://pronabes.sev.gob.mx/

Ministerio de Educación (2011). Resultados de la Información Estadística de Instituciones de Educación Superior. San Salvador. 